\title{
Separating minimal valuations, point-continuous valuations and continuous valuations
}

\author{
Jean Goubault-Larrecq ${ }^{* 1}$ and Xiaodong Jia ${ }^{\dagger 2}$ \\ ${ }^{1}$ Université Paris-Saclay, CNRS, ENS Paris-Saclay, Laboratoire \\ Méthodes Formelles, 91190, Gif-sur-Yvette, France \\ ${ }^{2}$ School of Mathematics, Hunan University, Changsha, Hunan, \\ 410082, China
}

September 2, 2021

\begin{abstract}
We give two concrete examples of continuous valuations on dcpo's to separate minimal valuations, point-continuous valuations and continuous valuations:

1. Let $\mathcal{J}$ be the Johnstone's non-sober dcpo, and $\mu$ be the continuous valuation on $\mathcal{J}$ with $\mu(U)=1$ for nonempty Scott opens $U$ and $\mu(U)=0$ for $U=\emptyset$. Then $\mu$ is a point-continuous valuation on $\mathcal{J}$ that is not minimal.

2. Lebesgue measure extends to a measure on the Sorgenfrey line $\mathbb{R}_{\ell}$. Its restriction to the open subsets of $\mathbb{R}_{\ell}$ is a continuous valuation $\lambda$. Then its image valuation $\bar{\lambda}$ through the embedding of $\mathbb{R}_{\ell}$ into its Smyth powerdomain $\mathcal{Q} \mathbb{R}_{\ell}$ in the Scott topology is a continuous valuation that is not point-continuous.
\end{abstract}

We believe that our construction $\bar{\lambda}$ might be useful in giving counterexamples displaying the failure of the general Fubini-type equations on dcpo's.

${ }^{*}$ Email: goubault@lsv.fr

${ }^{\dagger}$ Email (corresponding author): jiaxiaodong@hnu.edu.cn 


\section{Introduction}

Continuous valuations on topological spaces are analogues of measures on measurable spaces. In domain theory, continuous valuations on dcpo's with the Scott topology are employed by computer scientists and mathematicians to give denotational meanings to probabilistic programming languages. This line of work dates back to Jones and Plotkin [19, 18]. Indeed, in her Ph.D. thesis, Jones developed the theory of valuations and used the valuations monad $\mathcal{V}$ on the category DCPO of dcpo's and Scott-continuous maps to give denotational semantics to probabilistic programming languages.

While the valuations monad on the category DCPO enjoys many nice properties, for example this monad is a strong monad and dcpo-enriched, it is unknown whether it is a commutative monad on the same category. As a result, it would be difficult, using the valuations monad, to establish the socalled contextual equivalence between programs that only differ in the order of sampling random variables. To combat this problem, the authors in [17] constructed submonads of the valuations monad $\mathcal{V}$ that are commutative on the category of dcpo's. Among their construction, there is a least submonad of the valuations monad that consists of which we call minimal valuations. Minimal valuations are these continuous valuations that are in the d-closure of the simple valuations; precisely, they consist of directed suprema of simple valuations, directed suprema of directed suprema of simple valuations and so forth, transfinitely. Every minimal valuation is a point-continuous valuation in the sense of Heckmann [12, 17]. Heckmann[12] proved that the class of point-continuous valuations on space $X$ form the sobrification of the space of simple valuations on $X$, both in the so-called weak topology. Every pointcontinuous valuation is a continuous valuation [11, Proposition 3.1].

It is relatively easy to see that on general topological spaces minimal valuations form a strictly smaller class than that of point-continuous valuations. However, it is unknown whether the same is true on dcpo's with the Scott topology. The first example in this paper clarifies the difference between minimal valuations and point-continuous valuations on dcpo's. Concretely, we consider the well-known Johnstone's non-sober dcpo $\mathcal{J}$ and the "constant-1 valuation" $\mu$ on $\mathcal{J}$ defined by $\mu(U)=1$ if $U$ is nonempty and $\mu(\emptyset)=0$. We show that every bounded continuous valuation on $\mathcal{J}$ can be written as a sum of some discrete valuation and a scalar multiple of $\mu$. This enables us to conclude that every continuous valuation on $\mathcal{J}$ is actually point-continuous. Moreover, we prove that the continuous valuation $\mu$ is not in the d-closure of simple valuations, hence it serves as an example that separates minimal valuations from point-continuous valuations. This 
example is included in Section 3 .

Similar to the difference between minimal valuations and point-continuous valuations, continuous valuations that are not point-continuous can be easily found on topological spaces. For example, Lebesgue measure, restricted to the usual opens of reals, is a continuous valuation that is not pointcontinuous. However, it has been unknown whether point-continuous valuations differ from continuous valuation on dcpo's since 1996. The second goal of this note is to give an example of a continuous valuation on a $d c p o$ that is not point-continuous. In order to find such an example, one is tempted to find the simplest possible example, and typically to find a continuous valuation that takes only two values, 0 or 1 , and hoping that it would not be point-continuous. However, we notice that such a strategy cannot work, as we will see in Section 4.1. Hence, we will have to work a bit more. We show how one can build certain continuous valuation on the Sorgenfrey line $\mathbb{R}_{\ell}$ in Section 4.2 , including one based on Lebesgue measure $\lambda$. We study the compact subsets of $\mathbb{R}_{\ell}$ in Section 4.3 , as a preparation to studying the dcpo $\mathcal{Q} \mathbb{R}_{\ell}$ of compact subsets of $\mathbb{R}_{\ell}$ under reverse inclusion, and showing that the natural map from $\mathbb{R}_{\ell}$ to $\mathcal{Q} \mathbb{R}_{\ell}$ is a subspace embedding in Section 4.4. We transport Lebesgue measure $\lambda$ along this embedding, and we will show that the resulting continuous valuation $\bar{\lambda}$ on $\mathcal{Q} \mathbb{R}_{\ell}$ fails to be point-continuous in Section 4.5. We believe that our construction $\bar{\lambda}$ might be useful in giving counterexamples displaying the failure of the general Fubini-type equations on dcpo's, which is a longstanding open problem in domain theory. More detailed discussion about this part is included in the concluding remarks.

\section{Preliminaries}

We use standard concepts and notations from topology, measure theory, and domain theory. The reader is referred to [1, 7, 6] for topology and domain theory, and to [22] for measure theory.

\section{$2.1 \quad$ Valuations}

On a topological space $X$, a valuation $\nu$ is a map from the set $\mathcal{O} X$ of opens of $X$ to the extended reals $\overline{\mathbb{R}}_{+}$, satisfying strictness $(\nu(\emptyset)=0)$, monotonicity $(U \subseteq V \Rightarrow \nu(U) \leq \nu(V))$ and modularity $(\nu(U)+\nu(V)=\nu(U \cup V)+\nu(U \cap$ $V)$ ). A valuation $\nu$ on $X$ is called continuous if it is Scott-continuous from $\mathcal{O} X$ to $\overline{\mathbb{R}}_{+}$, and it is called bounded if $\nu(X)<\infty$. Continuous valuations are ordered in the stochastic order: $\nu_{1} \leq \nu_{2}$ if and only if $\nu_{1}(U) \leq \nu_{2}(U)$ for all opens $U$ of $X$. The set of all continuous valuations on $X$, which we 
denote as $\mathcal{V} X$, is a dcpo in the stochastic order. Canonical examples of continuous valuations on $X$ include Dirac valuations $\delta_{x}$ for $x \in X$, where $\delta_{x}(U)=1$ if $x \in U$ and $\delta_{x}(U)=0$ if $x \notin U$. As a dcpo, $\mathcal{V} X$ is closed under suprema, it also is closed under scalar multiplication and sum, for $\nu_{i} \in \mathcal{V} X$ and $r_{i} \in\left[0, \infty\left[, i=1, \cdots, n\right.\right.$, the sum $\sum_{i=1}^{n} r_{i} \nu_{i}$ which is defined by $\left(\sum_{i=1}^{n} r_{i} \nu_{i}\right)(U)=\sum_{i=1}^{n} r_{i} \nu_{i}(U)$ also is in $\mathcal{V} X$. For $r_{i} \in\left[0, \infty\left[\right.\right.$ and $x_{i} \in X$, $i=1, \cdots, n$, the finite sum $\sum_{i=1}^{n} r_{i} \delta_{x_{i}}$ is called a simple valuation on $X$. The set of all simple valuations on $X$ is denoted by $\mathcal{S} X$. Valuations of the form $\sum_{i}^{\infty} r_{i} \delta_{x_{i}}=\sup _{n \in \mathbb{N}} \sum_{i=1}^{n} r_{i} \delta_{x_{i}}$ are called discrete valuations. The smallest sub-dcpo of $\mathcal{V} X$ that contains $\mathcal{S} X$ (hence all discrete valuations on $X)$ is denoted by $\mathcal{M} X$, and every valuations in $\mathcal{M} X$ is called minimal. It is easy to see that for each minimal valuation $\nu, \nu$ is either a simple valuation, or a directed supremum of simple valuations, or a directed supremum of directed suprema of simple valuations $\cdots$, transfinitely. A valuation $\nu$ on a space $X$ is point-continuous if and only if for every open subset $U$, for every real number $r$ such that $0 \leq r<\nu(U)$, there is a finite subset $A$ of $U$ such that $\nu(V)>r$ for every open neighborhood $V$ of $A$. Minimal valuations are point-continuous [17], and point-continuous valuations are continuous valuations [11, Proposition 3.1].

\section{$2.2 \quad$ Ring of sets}

We will need the notion of Boolean ring of sets (ring of sets for short). On a set $X$, a ring of sets on $X$ is a lattice of sets consisting of subsets of $X$ that also is closed under relative complements. For a topological space $X$, the set $\mathcal{O} X$ of all opens of $X$ is a lattice of sets, and the ring of sets generated by $\mathcal{O} X$ is the intersection of all rings of sets on $X$ that contain $\mathcal{O} X$, and it is denoted by $\mathcal{A}(\mathcal{O} X)$.

Lemma 2.1. [6, Lemma IV-9.2] Let $X$ be a topological space. For each set $A$ in $\mathcal{A}(\mathcal{O} X)$, the ring of sets generated by open sets of $X, A$ is of the form of a finite disjoint union $\coprod_{i=1}^{n} U_{i} \backslash V_{i}$, where $U_{i}$ and $V_{i}$ are open subsets of $X$. One can also stipulate that $V_{i} \subseteq U_{i}$ for each $i$.

For open subsets $U$ and $V$ of $X$, the set difference $U \backslash V$ is called a crescent. Since $U \backslash V=U \backslash(U \cap V)$, in the sequel, when we write a set $A \in \mathcal{A}(\mathcal{O} X)$ as $\coprod_{i=1}^{n} U_{i} \backslash V_{i}$, we always assume that $V_{i} \subseteq U_{i}$ for each $i$.

Lemma 2.2. [12, Section 3.3] Let $X$ be a topological space and $\mu$ be a bounded continuous valuation on $X$. For each set $A=\coprod_{i=1}^{n} U_{i} \backslash V_{i}$ in $\mathcal{A}(\mathcal{O} X)$, define $\mu_{A}=\left.\sum_{i=1}^{n} \mu\right|_{U_{i}} ^{V_{i}}$, where for open sets $U, V$ and $W,\left.\mu\right|_{U} ^{V}(W)=$ 
$\mu(W \cap U)-\mu(W \cap V \cap U)$. Then $\mu_{A}$ is a bounded continuous valuation for each $A \in \mathcal{A}(\mathcal{O} X)$. In particular, $\mu_{U \backslash V}=\left.\mu\right|_{U} ^{V}$. Note that $\mu_{U}=\left.\mu\right|_{U} ^{\emptyset}$ for every open subset $U$.

Note that it is possible that for each $A \in \mathcal{A}(\mathcal{O} X), A$ can be written as a disjoint union $\coprod_{i=1}^{n} U_{i} \backslash V_{i}$ or $\coprod_{j=1}^{m} U_{j} \backslash V_{j}$. However, when $\coprod_{i=1}^{n} U_{i} \backslash V_{i}=$ $\coprod_{i=j}^{m} U_{j} \backslash V_{j}$, we will always have that $\left.\sum_{i=1}^{n} \mu\right|_{U_{i}} ^{V_{i}}=\left.\sum_{j=1}^{m} \mu\right|_{U_{j}} ^{V_{j}}$. This validates the definition of $\mu_{A}$ in the previous lemma.

Lemma 2.3. For two disjoint sets $A, B \in \mathcal{A}(\mathcal{O} X), \mu_{A \cup B}=\mu_{A}+\mu_{B}$. This implies that $\mu_{A} \leq \mu_{B}$ when $A \subseteq B$.

Proof. From the above remark and straightforward computation.

Lemma 2.4. Let $X$ be a $T_{0}$ topological space and $\mu$ be a bounded continuous valuation on $X$. If $\{a\}$ is in $\mathcal{A}(\mathcal{O} X)$, then there exist opens $U, V$ with $V \subseteq U$ and $U \backslash V=\{a\}$. Moreover, in this case $\mu_{\{a\}}=r_{a} \delta_{a}$, where $r_{a}=\mu_{\{a\}}(X)=\mu(U)-\mu(V)=\mu_{\{a\}}(U)$.

Proof. The first assertion is obvious.

For the second assertion, since $X$ is $T_{0}$, we only need to prove that for each open $O, \mu_{a}(O)$ is equal to $r_{a}$ if $a \in O$ and to 0 if $a \notin O$. Assume that $U$ and $V$ are open subsets of $X$ with $V \subseteq U$ and $U \backslash V=\{a\}$. For an open subset $O$, if $a \in O$, then $O \cap U \backslash O \cap V=U \backslash V=\{a\}$, which implies that $\mu_{a}(O)=\left.\mu\right|_{U} ^{V}(O)=\left.\mu\right|_{U \cap O} ^{V \cap O}(O)=\left.\mu\right|_{U \cap O} ^{V \cap O}(U)=\mu_{a}(U)$. If $a$ is not in $O$, then $O \cap U=O \cap V$. Hence $\mu_{a}(O)=0$.

\section{Point-continuous valuations need not be mini- mal valuations}

In this section, we built a point-continuous valuation $\mu$ on the well-known Johnstone's non-sober dcpo, which is not a minimal valuation.

\subsection{Valuations on $\mathbb{N}_{\text {cof }}$}

Let $\mathbb{N}_{\text {cof }}$ be the topological space of natural numbers equipped with the cofinite topology. It is easy to verity that the the map $\beta: \mathcal{O N}$ cof $\rightarrow \overline{\mathbb{R}}_{+}$defined by

$$
\beta(U)= \begin{cases}1, & U \subseteq \mathbb{N}_{\text {cof }} \text { is open and nonempty } \\ 0, & U \text { is empty }\end{cases}
$$

is a bounded continuous valuation on $\mathbb{N}_{\text {cof }}$. 
Lemma 3.1. For each $i \in \mathbb{N}_{\text {cof }}$, the set $\{i\}$ is closed. Hence it is in the ring of sets generated by the co-finite topology on $\mathbb{N}_{\text {cof }}$.

Proof. Straightforward.

Proposition 3.2. Let $\nu$ be a bounded continuous valuation on $\mathbb{N}_{\text {cof }}$. Then there exists a discrete valuation $\alpha$ on $\mathbb{N}_{\text {cof }}$ and nonnegative real number $r$ such that $\nu=\alpha+r \beta$.

Proof. We let $\alpha=\sum_{i \in \mathbb{N}} \nu_{\{i\}}$. By Lemma 3.1 and Lemma 2.2, each $\nu_{\{i\}}$ is a continuous valuation. Since $\mathbb{N}_{\text {cof }}$ is a $T_{0}$ topological space, it follows from Lemma 2.4 that $\alpha$ is a discrete valuation. Now we define the map

$$
\nu^{*}: \mathcal{O} \mathbb{N}_{\text {cof }} \rightarrow \mathbb{R}_{+}:: U \mapsto \nu(U)-\alpha(U) .
$$

We proceed to show that $\nu^{*}$ is a multiple of $\beta$, that is, there exists some $r \in\left[0, \infty\left[\right.\right.$ such that $\nu^{*}=r \beta$.

First, for each $n \in \mathbb{N}, \nu(U)-\sum_{i=1}^{n} \nu_{\{i\}}(U)=\nu_{\mathbb{N} \backslash\{1,2, \ldots, n\}}(U)$ is nonnegative, hence $\nu^{*}(U)$, which is the infimum of $\nu(U)-\sum_{i=1}^{n} \nu_{\{i\}}(U), n \in \mathbb{N}$, indeed takes values in $\mathbb{R}_{+}$.

Second, for nonempty open sets $U$ and $V$ with $V \subseteq U$, we prove that $\nu^{*}(U)=\nu^{*}(V)$. Because $V$ is co-finite, we know that $U \backslash V$ is a finite set, which we denote by $F$. Then we know

$$
\begin{array}{rlr}
\nu^{*}(U) & =\nu(U)-\sum_{i=1}^{\infty} \nu_{\{i\}}(U) & \text { definition of } \nu^{*} \\
& =\nu_{U}(U)-\sum_{i=1}^{\infty} \nu_{\{i\}}(U) & \nu_{U}(U)=\nu(U \cap U) \\
& =\nu_{V}(U)+\sum_{i \in F} \nu_{\{i\}}(U)-\sum_{i=1}^{\infty} \nu_{\{i\}}(U) & \text { by Lemma } 2.3 \text { and } V \cup F=U \\
& =\nu_{V}(V)-\sum_{i=1}^{\infty} \nu_{\{i\}}(V) & \text { by Lemma } 2.4 \\
& =\nu^{*}(V) . & \text { definition of } \nu^{*}
\end{array}
$$

Now for general nonempty opens $U$ and $V$, we have $\nu^{*}(U)=\nu^{*}(U \cap V)=$ $\nu^{*}(V)$. Let $r=\nu^{*}(X)$, then we know that $r$ is nonnegative from above and that $\nu^{*}=r \beta$ (hence $\nu^{*}$ also is a continuous valuation).

Finally, we conclude the proof by the fact that $\nu=\alpha+\nu^{*}=\alpha+r \beta$. 


\subsection{Valuations on Johnstone's non-sober dcpo $\mathcal{J}$}

In 1980, Johnstone gave the first dcpo which is not sober in the Scott topology [20]. This dcpo, which we denote by $\mathcal{J}$, serves as a basic building block in several counterexamples in domain theory [15, 14]. In this subsection, we will use it to construct a continuous valuation that is not minimal.

Definition 3.3 (The dcpo $\mathcal{J}$ ). Let $\mathbb{N}$ be the set of natural numbers and $\mathcal{J}=\mathbb{N} \times(\mathbb{N} \cup\{\infty\})$. The order on $\mathcal{J}$ is defined by $(a, b) \leq(c, d)$ if and only if either $a=c$ and $b \leq d$, or $d=\infty$ and $b \leq c$.

The structure of $\mathcal{J}$ is depicted in Figure 1.

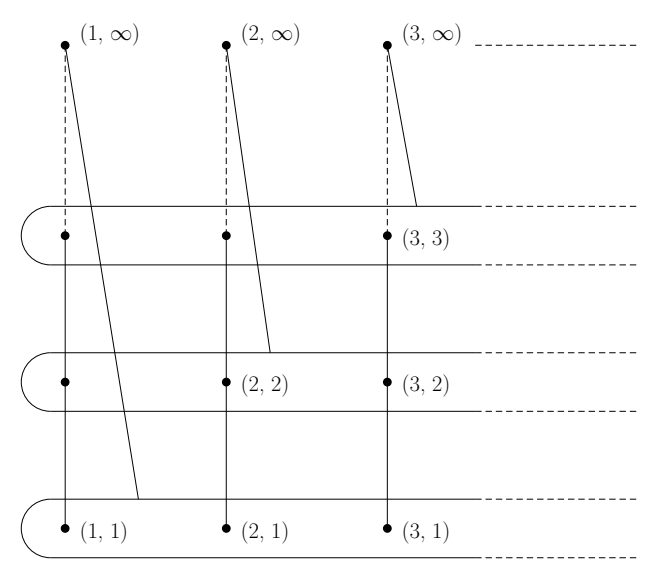

Figure 1: Johnstone's non-sober dcpo $\mathcal{J}$.

We will use the following convention throughout this subsection when we reason about $\mathcal{J}$.

- $M$ denotes the set of all maximal points of $\mathcal{J}$, that is $M=\{(i, \infty) \mid$ $i \in \mathbb{N}\}$;

- $M_{k}=\{(i, \infty) \mid k<i\}$, and $M_{k, l}=\{(i, \infty) \mid k<i \leq l\}$ for $k, l \in \mathbb{N}$ and $k<l$;

- $N$ denotes the set $\mathcal{J} \backslash M$; elements in $N$ are of finite height;

- $L_{i}$ denotes the set of points of $\mathcal{J}$ which are at Level $i$, for each $i \in \mathbb{N}$, that is $L_{i}=\{(j, i) \mid j \in \mathbb{N}\}$; 
- $C_{i}$ denotes the set of points of $\mathcal{J}$ which are in Column $i$, that is $C_{i}=\{(i, j) \mid j \in \mathbb{N} \cup\{\infty\}\}$.

- $D_{i}$ denotes the set $\bigcup_{j \leq i} C_{j}$, i.e., $D_{i}$ consists of elements in the first $i$-many columns.

The dcpo $\mathcal{J}$ in the Scott topology is a non-sober topological space, and the set $M$ of maximal points of $\mathcal{J}$ equipped with the relative Scott topology is homeomorphic to $\mathbb{N}_{\text {cof }}$.

Let $\nu$ be an arbitrarybounded continuous valuation on $\mathcal{J}$. We are going to show that $\nu$ can be written as a sum of a discrete valuation $\theta$ and $r \mu$, where $r$ is a nonnegative real number and in this section, $\mu$ is reserved for the fixed valuation on $\mathcal{J}$ that takes value 1 on nonempty Scott-opens, and 0 on the empty set:

$$
\mu(U)= \begin{cases}1, & U \neq \emptyset \\ 0, & U=\emptyset .\end{cases}
$$

Note that $\mu$ on $\mathcal{J}$ is an analogue of $\beta$ on $\mathbb{N}_{\text {cof }}$. Indeed, the pushforward image of $\beta$ along the canonical topological embedding $n \mapsto(n, \infty)$ of $\mathbb{N}_{\text {cof }}$ into $\mathcal{J}$ is exactly the valuation $\mu$. Since $\theta$ and $r \mu$ are point-continuous (direct verification), by proving that $\nu$ is a sum of some discrete $\theta$ and $r \mu$, we infer that all (not necessarily bounded) continuous valuations on $\mathcal{J}$ are point-continuous by using a trick due to Heckmann.

Theorem 3.4. Every bounded continuous valuation $\nu$ on $\mathcal{J}$ is point-continuous. Moreover, there exist a discrete valuation $\theta$ and nonnegative real number $r$ such that $\nu=\theta+r \mu$.

We prove this theorem by a series of results. First, we give a lemma that will be used a few times. It is a slight generalisation of [12, Proposition 3.2].

Lemma 3.5. Let $\mu$ be a bounded continuous valuation on a space $X$, and $\nu$ be a monotonic map from $\mathcal{O} X$ to $\mathbb{R} \cup\{-\infty,+\infty\}$ such that $\nu(\emptyset)=0$. If $\mu+\nu$ is a continuous valuation, then so is $\nu$.

Proof. Since $\nu$ is monotonic and $\nu(\emptyset)=0, \nu$ actually takes its values in $\overline{\mathbb{R}}_{+}$. It is clear that $\nu=(\mu+\nu)-\mu$ is modular, using the fact that $\mu$ is bounded for the subtraction to make sense. The only challenge is Scott-continuity. Let $\left(U_{i}\right)_{i \in I}$ be any directed family of open subsets of $X$, and $U$ be its union. We have $\nu(U) \geq \sup _{i \in I}^{\uparrow} \nu\left(U_{i}\right)$ by monotonicity. In order to prove the reverse inequality, we consider any $a<\nu(U)$, and we show that $a \leq \nu\left(U_{i}\right)$ for some $i \in I$. Since $\mu$ is bounded, $\mu(U)+a<(\mu+\nu)(U)$, and since $\mu+\nu$ is a 
continuous valuation, there is an $i \in I$ such that $\mu(U)+a<(\mu+\nu)\left(U_{i}\right)$. Then $a<\mu\left(U_{i}\right)-\mu(U)+\nu\left(U_{i}\right) \leq \nu\left(U_{i}\right)$.

Lemma 3.6. For each element $a \in N$ (using the convention after Definition 3.3), the singleton $\{a\}$ is a crescent. Thus, for each $a \in N,\{a\}$ in the ring of sets generated by Scott-opens of $\mathcal{J}$.

Proof. For each $a \in N$, if $a$ is at Level $n$, that is $a=(j, n)$ for some $j \in \mathbb{N}$, then $\{a\}$ can be written as $\left(\{a\} \cup \uparrow L_{n+1}\right) \backslash \uparrow L_{n+1}$. The proof is done since both $\{a\} \cup \uparrow L_{n+1}$ and $\uparrow L_{n+1}$ are Scott-open in $\mathcal{J}$.

Proposition 3.7. For each Scott-open subset $U$ of $\mathcal{J}$, let $\nu^{*}(U)=\nu(U)-$ $\sum_{a \in N} \nu_{\{a\}}(U)$. Then $\nu^{*}$ is a bounded continuous valuation on $\mathcal{J}$.

Proof. Since for each $a \in N,\{a\}$ is a crescent by Lemma 3.6. $\nu_{\{a\}}$ is a continuous valuation. Hence $\nu_{\{a\}}(U)$ makes sense for each $a \in N$.

By Lemma 3.5, we only need to prove that $\nu^{*}$ is well-defined and orderpreserving. Note that $N$ is a countable set. We index elements in $N$ by natural numbers by letting $N=\left\{a_{1}, a_{2}, \ldots, a_{n}, \ldots\right\}$. Since for each open set $U, \sum_{i=1}^{n} \nu_{\left\{a_{i}\right\}}(U)=\nu_{\left\{a_{1}, \ldots, a_{n}\right\}}(U) \leq \nu_{X}(U)=\nu(U)$ and $\nu(U)$ is bounded, it means that for each $U$ the sequence $\nu_{\left\{a_{i}\right\}}(U), n=1, \ldots, n, \ldots$ is commutatively summable. Hence $\nu^{*}$ is well-defined and takes values in $\mathbb{R}_{+}$.

For monotonicity of $\nu^{*}$, we let $U$ be Scott-open and compute as follows:

$$
\begin{aligned}
\nu^{*}(U) & =\nu(U)-\sum_{a \in N} \nu_{\{a\}}(U) \\
& =\nu(U)-\sum_{i=1}^{\infty} \nu_{\left\{a_{i}\right\}}(U) \\
& =\lim _{n \rightarrow \infty}\left(\nu(U)-\sum_{i=1}^{n} \nu_{\left\{a_{i}\right\}}(U)\right) \\
& =\inf _{n \in \mathbb{N}}\left(\nu_{X}(U)-\nu_{\left\{a_{1}, \ldots, a_{n}\right\}}(U)\right) \\
& =\inf _{n \in \mathbb{N}} \nu_{X \backslash\left\{a_{1}, \ldots, a_{n}\right\}}(U) .
\end{aligned}
$$

Since for each $n \in \mathbb{N}, \nu_{X \backslash\left\{a_{1}, \ldots, a_{n}\right\}}$ is a continuous valuation therefore a nonnegative order-preserving map. Hence the pointwise infimum $\nu^{*}$ of $\nu_{X \backslash\left\{a_{1}, \ldots, a_{n}\right\}}, n \in$ $\mathbb{N}$ also is order-preserving.

Lemma 3.8. For any two Scott-open subsets $U, V$ of $\mathcal{J}$ with $M \cap U=M \cap V$, $\nu^{*}(U)=\nu^{*}(V)$. 
Proof. Without loss of generality, we assume that $U \subseteq V$. Let $V_{n}=U \cup$ $\left(V \cap D_{n}\right)$ (see the convention after Definition 3.3). Since $M \cap U=M \cap V$, $V_{n}$ is Scott-open for each $n$. Moreover, $V=\bigcup_{n=1}^{\infty} V_{n}$.

Note that for every $n, V_{n} \backslash U=\left(V \cap D_{n}\right) \backslash U$ is a finite subset of $N$, again by the fact that $M \cap U=M \cap V$. Then we know that

$$
\begin{aligned}
\nu^{*}\left(V_{n}\right) & =\nu^{*}(U)+\nu^{*}\left(V_{n}\right)-\nu^{*}(U) \\
& =\nu^{*}(U)+\left(\nu\left(V_{n}\right)-\nu(U)\right)-\left(\sum_{a \in N} \nu_{\{a\}}\left(V_{n}\right)-\sum_{a \in N} \nu_{\{a\}}(U)\right) \\
& =\nu^{*}(U)+\nu_{V_{n} \backslash U}\left(V_{n}\right)-\left(\sum_{a \in N} \nu_{\{a\}}\left(V_{n}\right)-\sum_{a \in N} \nu_{\{a\}}(U)\right) \\
& =\nu^{*}(U)+\sum_{a \in V_{n} \backslash U} \nu_{\{a\}}\left(V_{n}\right)-\sum_{a \in N \cap\left(V_{n} \backslash U\right)} \nu_{\{a\}}\left(V_{n}\right) \\
& =\nu^{*}(U) .
\end{aligned}
$$

Hence by Scott-continuity of $\nu^{*}$ (Proposition 3.7), we know that $\nu^{*}(V)=$ $\nu^{*}\left(\bigcup_{n \in \mathbb{N}} V_{n}\right)=\sup _{n \in \mathbb{N}} \nu^{*}\left(V_{n}\right)=\nu^{*}(U)$.

We consider $M$ as a subspace of $\mathcal{J}$ with the Scott-topology, and define a continuous valuation $\nu^{\infty}$ on $M$ by stipulating for each open subset $W$ of $M$ that $\nu^{\infty}(W)=\nu^{*}\left(U_{W}\right)$, where $U_{W}$ is the largest Scott-open subset of $\mathcal{J}$ with $M \cap U_{W}=W$. We now use Lemma 3.8 to prove that $\nu^{\infty}$ is indeed a bounded continuous valuation on $M$. This is a consequence of [9, Proposition 5.2], we provide a direct proof here nevertheless.

Lemma 3.9. The map $\nu^{\infty}$ is a bounded continuous valuation on $M$ equipped with the relative $S$ cott topology from $\mathcal{J}$.

Proof. It is easy to see that $\nu^{\infty}(\emptyset)=0$.

For modularity, let $W_{1}$ and $W_{2}$ be two open subsets of $M$. Then we have:

$$
\begin{array}{rlrl}
\nu^{\infty}\left(W_{1}\right)+\nu^{\infty}\left(W_{2}\right) & =\nu^{*}\left(U_{W_{1}}\right)+\nu^{*}\left(U_{W_{2}}\right) & & \text { definition of } \nu^{\infty} \\
& =\nu^{*}\left(U_{W_{1}} \cup U_{W_{2}}\right)+\nu^{*}\left(U_{W_{1}} \cap U_{W_{2}}\right) & \nu^{*} \text { is a valuation } \\
& =\nu^{*}\left(U_{W_{1} \cup W_{2}}\right)+\nu^{*}\left(U_{W_{1} \cap W_{2}}\right) & & \text { by Lemma 3.8 } \\
& =\nu^{\infty}\left(W_{1} \cup W_{2}\right)+\nu^{\infty}\left(W_{1} \cap W_{2}\right) . & & \text { definition of } \nu^{\infty}
\end{array}
$$

Now we prove that $\nu^{\infty}$ is Scott-continuous. Let $W_{i}, i \in I$ be a directed family of open subsets of $M$ and $W=\bigcup_{i \in I} W_{i}$. We first note that $U_{W_{i}}, i \in I$ also 
form a directed family of Scott-opens in $\mathcal{J}$. Hence we have

$$
\begin{array}{rlr}
\nu^{\infty}(W) & =\nu^{*}\left(U_{\bigcup_{i \in I} W_{i}}\right) & \text { definition of } \nu^{\infty} \\
& =\nu^{*}\left(\bigcup_{i \in I} U_{W_{i}}\right) & \text { by Lemma 3.8 } \\
& =\sup _{i \in I} \nu^{*}\left(U_{W_{i}}\right) & \nu^{*} \text { is Scott-continuous } \\
& =\sup _{i \in I} \nu^{\infty}\left(W_{i}\right) . & \text { definition of } \nu^{\infty}
\end{array}
$$

Finally, boundedness of $\nu^{\infty}$ is clear since $\nu^{*}$ is.

We are now ready to prove Theorem 3.4 .

Proof of Theorem 3.4. The space $M$ in the relative Scott topology from $\mathcal{J}$ is homeomorphic to $\mathbb{N}_{\text {cof }}$. For example, one of the (infinitely many) homeomorphisms between them is the map sending $n \in \mathbb{N}_{\text {cof }}$ to $(n, \infty) \in M$. It then follows from Proposition 3.2 that $\nu^{\infty}$ can be written as $\alpha+r \beta$ where $\alpha$ is a discrete valuation on $M, r$ is a nonnegative real number, and $\beta$ is the valuation on $M$ defined as $\beta(W)=1$ if $W \subseteq M$ is nonempty, and $\beta(W)=0$ if $W=\emptyset$. For each Scott open subset $U$ of $\mathcal{J}$, we have

$$
\nu^{*}(U)=\nu^{\infty}(U \cap M)=\alpha(U \cap M)+r \beta(U \cap M) .
$$

Now we define $\theta^{\prime}(U)=\alpha(U \cap M)$. Since $\alpha$ is a discrete valuation on $M$, it is obvious that $\theta^{\prime}$ is a discrete valuation on $\mathcal{J}$. Hence, by combining Proposition 3.7 and the fact that $\mu(U)=\beta(U \cap M)$, we know that for each Scott open subset $U$ of $\mathcal{J}$,

$$
\nu(U)=\sum_{a \in N} \nu_{\{a\}}(U)+\nu^{*}(U)=\sum_{a \in N} \nu_{\{a\}}(U)+\theta^{\prime}(U)+r \mu(U) .
$$

Finally, we let $\theta=\theta^{\prime}+\sum_{a \in N} \nu_{\{a\}}$ and the proof is complete.

As a corollary to Theorem 3.4, we have the following:

Corollary 3.10. Every continuous valuation on $\mathcal{J}$ is point-continuous.

Proof. It is proved in [12, Theorem 4.2] that every continuous valuation on a $T_{0}$ topological space can be written as a directed supremum of bounded continuous valuations. By Theorem 3.4, we know that on $\mathcal{J}$ every bounded continuous valuation is of the form $\theta+r \mu$, which is obviously point-continuous. Hence every continuous valuation on $\mathcal{J}$, as a directed supremum of pointcontinuous valuations, also is point-continuous [12, Section 3.2, Item (5)]. 


\section{$3.3 \quad$ Non-minimality of $\mu$ on $\mathcal{J}$}

In this subsection, we show that the valuation $\mu$ on $\mathcal{J}$, defined in the last subsection, is not a minimal valuation.

As we know from Theorem 3.4, every bounded continuous valuation $\nu$ on $\mathcal{J}$ is of the form $\theta+r \mu$. We first prove that that for any $r>0, \theta+r \mu$ can not be written as a supremum of discrete valuations. Without loss of generality, in the sequel we assume that the total mass of $\theta+r \mu$ is 1 , i.e., $\theta(\mathcal{J})+r \mu(\mathcal{J})=\theta(\mathcal{J})+r=1$.

We will need the following extension result for discrete valuations, which is a special case of [3], Theorem 4.1].

Lemma 3.11. Let $\theta$ be a discrete valuation on a dcpo $D$ with $\theta(D)<\infty$. Then $\theta$ has a unique extension to a measure, which we again denote as $\theta$, on the Borel $\sigma$-algebra of $(D, \sigma D)$, where $\sigma D$ is the Scott topology on $D$. In fact, if $\theta=\sum_{i \in \mathbb{N}} r_{i} \delta_{x_{i}}$ with $\sum_{i \in \mathbb{N}} r_{i}<\infty$, then the value of its measure extension on a Borel subset $B$ of $D$ is just $\sum_{i / x_{i} \in B} r_{i}$.

Lemma 3.6 says that for each $a \in N \subseteq \mathcal{J}$, the singleton $\{a\}$ is a crescent, hence $\{a\}$ is a Borel subset of $\mathcal{J}$. Actually, every subset of $\mathcal{J}$ is a Borel subset. To see this, we only need to show that $\{(i, \infty)\}$ is a Borel subset in $\mathcal{J}$ for each $i \in \mathbb{N}$, since $\mathcal{J}$ is countable. Indeed, $\{(i, \infty)\}=\downarrow(i, \infty) \backslash \bigcup_{i \in \mathbb{N}} \downarrow L_{j}$, and for $i, j \in \mathbb{N}, \downarrow(i, \infty)$ and $\downarrow L_{j}$ are Scott-closed. Now if $\theta$ is a discrete valuation on $\mathcal{J}$, then it makes sense to apply $\theta$ to any subset of $\mathcal{J}$, viewing that $\theta$ is a measure defined on the Borel $\sigma$-algebra generated by Scott-opens on $\mathcal{J}$.

Proposition 3.12. Let $\theta$ be a discrete valuation on $\mathcal{J}$ with total mass $1-r$ and $r>0$. Then $\theta+r \mu$ is not a directed supremum of discrete valuations.

Proof. Suppose, for the sake of contradiction, that there exists a directed family $\left\{\theta_{a}\right\}_{a \in A}$ of discrete valuations with supremum $\sup _{a \in A} \theta_{a}=\theta+r \mu$.

Since $(\theta+r \mu)(\mathcal{J})=1$, there exists a discrete valuation $\theta_{a}, a \in A$ such that $\theta_{a}(\mathcal{J})>1-\frac{r}{4}$. Note that $\sup _{i \in \mathbb{N}} D_{i}=\mathcal{J}$, where $D_{i}=\bigcup_{j \leq i} C_{j}$ consists of points in the first $i$-many columns (see Section 3.2), and also that $D_{i}$ is a Borel subset of $\mathcal{J}$ for each $i \in \mathbb{N}$. Since $\theta_{a}$ extends to a measure (Lemma 3.11), we know that there exists a $k \in \mathbb{N}$ such that $\theta_{a}\left(D_{k}\right)>1-\frac{r}{4}$.

Obviously, $\downarrow D_{k}$ is Scott-closed and $U_{k}=\mathcal{J} \backslash \downarrow D_{k}$ is a nonempty Scottopen subset of $\mathcal{J}$, hence $(\theta+r \mu)\left(U_{k}\right) \geq r>\frac{3 r}{4}$. Again since $\theta+r \mu=$ $\sup _{a \in A} \theta_{a}$, there exists $\theta_{b}>\theta_{a}$ such that $\theta_{b}\left(U_{k}\right)>\frac{3 r}{4}$.

Note that the set $\uparrow D_{k}$ is a filtered intersection of countably many Scottopen subsets of $\mathcal{J}$, for example one can write $\uparrow D_{k}=\bigcap_{i \in \mathbb{N}} O_{i}$, where for 
each $i, O_{i}=\mathcal{J} \backslash \downarrow\{(k+1, i),(k+2, i), \ldots\}$. Then we know that

$$
\theta_{b}\left(\uparrow D_{k}\right)=\inf _{i \in \mathbb{N}} \theta_{b}\left(O_{i}\right) \geq \inf _{i \in \mathbb{N}} \theta_{a}\left(O_{i}\right)=\theta_{a}\left(\uparrow D_{k}\right) \geq \theta_{a}\left(D_{k}\right)>1-\frac{r}{4} .
$$

Since $M_{k}=\{(i, \infty) \mid k<i\}$ is a Borel subset of $\mathcal{J}$ and it equals to $\uparrow D_{k} \cap U_{k}$, hence by inclusion-exclusion of $\theta_{b}$ we know

$$
\theta_{b}\left(M_{k}\right)+\theta_{b}(\mathcal{J}) \geq \theta_{b}\left(U_{k}\right)+\theta_{b}\left(\uparrow D_{k}\right)>1+\frac{r}{2},
$$

from which it follows that $\theta_{b}\left(M_{k}\right)>\frac{r}{2}$. Since $\bigcup_{l / l>k} M_{k, l}=M_{k}\left(M_{k, l}=\right.$ $\{(i, \infty) \mid k<i \leq l\})$, there exists a big enough $l>k$ such that $\theta_{b}\left(M_{k, l}\right)>\frac{r}{2}$.

Now consider $U_{l}=\mathcal{J} \backslash \downarrow D_{l}$. Since $(\theta+r \mu)\left(U_{l}\right) \geq r>\frac{3 r}{4}$, we find a $\theta_{c}, c \in A$ such that $\theta_{c} \geq \theta_{b}$ and $\theta_{c}\left(U_{l}\right)>\frac{3 r}{4}$. Meanwhile, note that $D_{k} \subseteq$ $D_{l}$, similar to the reasoning above we will have that $\theta_{c}\left(\uparrow D_{l}\right) \geq \theta_{b}\left(\uparrow D_{l}\right) \geq$ $\theta_{b}\left(\uparrow D_{k}\right)>1-\frac{r}{4}$ and that $\theta_{c}\left(M_{l}\right)>\frac{r}{2}$. Then there exists a large enough natural number $m>l$ such that $\theta_{c}\left(M_{l, m}\right)>\frac{r}{2}$.

We claim that $\theta_{c}(\mathcal{J})>r$. Indeed, $\theta_{c}(\mathcal{J}) \geq \theta_{c}\left(M_{k, m}\right)=\theta_{c}\left(M_{k, l}\right)+$ $\theta_{c}\left(M_{l, m}\right) \geq \theta_{b}\left(M_{k, l}\right)+\theta_{c}\left(M_{l, m}\right)>r$. The second to last inequality comes from the fact that $\theta_{c} \geq \theta_{b}$ and $M_{k, l}$ is a filtered intersection of countably many Scott-open subsets of $\mathcal{J}$ : one may take such a filtered family as $\{\mathcal{J} \backslash$ $\left.\downarrow F \mid F \subseteq_{\text {fin }} M \backslash M_{k, l}\right\}$.

Next we consider the Scott-open set $U_{m}=\mathcal{J} \backslash \downarrow D_{m}$ and then find $\theta_{d} \geq \theta_{c}$ with $\theta_{d}(\mathcal{J})>\frac{3 r}{2}$, and so forth. We proceed the above process $N$ times, where $N$ is a natural number satisfying that $N \times \frac{r}{2}>1$, to find a discrete valuation $\theta_{z}$ in the directed family $\left\{\theta_{a}\right\}_{a \in A}$. By the construction, we would know that $\theta_{z}(\mathcal{J})>1$. However, this is impossible since $\theta_{z} \leq \theta+r \mu$ and $(\theta+r \mu)(\mathcal{J})=1$. So our assumption of the existence of the directed family $\left\{\theta_{a}\right\}_{a \in A}$ of discrete valuations with $\sup _{a \in A} \theta_{a}=\theta+r \mu$ mush have been wrong, and we finish the proof.

Theorem 3.13. Let $\theta$ be a discrete valuation on $\mathcal{J}$ with total mass $1-r$ and $r>0$. Then $\theta+r \mu$ is not a minimal valuation.

Proof. We know that the set of minimal valuations (bounded by 1 ) on $\mathcal{J}$ is obtained by taking directed suprema of simple valuations (bounded by 1), directed suprema of directed suprema of simple valuations (bounded by 1), and so forth, transfinitely. However, from Theorem 3.4 and Proposition 3.12, at each of these steps we only obtain discrete valuations. Hence, by transfinite induction $\theta+r \mu$ is not a minimal valuation for $r>0$.

The following result, as promised, is then obvious:

Corollary 3.14. The valuation $\mu$ on $\mathcal{J}$ is point-continuous but not minimal. 


\section{Continuous valuations need not be point-continuous}

In the last section we have seen that on dcpo's not all point-continuous valuations are minimal. In this section, we present another separation result: on dcpo's, not all continuous valuations are point-continuous, a result which is known on general topological spaces, but unknown on dcpo's with the Scott topology.

\subsection{What are non-point-continuous valuations like?}

Tix showed that, on a sober space $X$, the bounded continuous valuations that take only finitely many values are the simple valuations, namely the finite linear combinations $\sum_{i=1}^{n} a_{i} \delta_{x_{i}}$, where each coefficient $a_{i}$ is in $\mathbb{R}_{+}$and $x_{i} \in X$ [24, Satz 2.2]. Hence they are all point-continuous.

We will extend that result slightly in this subsection. We first recall that a nonempty subset $A$ of a space $X$ is irreducible if $A \subseteq B \cup C$, for closed subsets $B$ and $C$ of $X$, implies that $A \subseteq B$ or $A \subseteq C$. $X$ is sober if every irreducible closed subset $C$ of $X$ is the closure of some unique singleton subset of $X$.

Lemma 4.1. Let $X$ be a topological space. For every irreducible closed subset $C$ of $X$, let $\mathfrak{e}_{C}: \mathcal{O} X \rightarrow \overline{\mathbb{R}}_{+}$map every open subset $U$ of $X$ to 1 if $U$ intersects $C$, to 0 otherwise. Then $\mathfrak{e}_{C}$ is a point-continuous valuation. If $C=\downarrow x$ for some point $x$, then $\mathfrak{e}_{C}=\delta_{x}$.

Proof. It is clear that $\mathfrak{e}_{C}$ is strict. In order to show point-continuity, we assume $0 \leq r<\mathfrak{e}_{C}(U)$. We note that $C$ must intersect $U$, say at $x$, and that

$0 \leq r<1$. Let $A \stackrel{\text { def }}{=}\{x\}$. For every open neighborhood $V$ of $A, V$ intersects $C($ at $x)$, so $\mathfrak{e}_{C}(V)=1>r$.

In order to show modularity, we observe that, since $C$ is irreducible, for all open subsets $U$ and $V$ of $X, C$ intersects both $U$ and $V$ if and only if $C$ intersects $U \cap V$. Hence $\mathfrak{e}_{C}(U \cap V)=\min \left(\mathfrak{e}_{C}(U), \mathfrak{e}_{C}(V)\right)$. Since $C$ intersects $U$ or $V$ if and only if $C$ intersects $U \cup V$, we have $\mathfrak{e}_{C}(U \cup$ $V)=\max \left(\mathfrak{e}_{C}(U), \mathfrak{e}_{C}(V)\right)$. Now for all real numbers $a$ and $b, \max (a, b)+$ $\min (a, b)=a+b$, so $\mathfrak{e}_{C}(U \cap V)+\mathfrak{e}_{C}(U \cup V)=\mathfrak{e}_{C}(U)+\mathfrak{e}_{C}(V)$.

When $C=\downarrow x$, for every $U \in \mathcal{O} X$ we have $\mathfrak{e}_{C}(U)=1$ if and only if $C$ intersects $U$, if and only if $x \in U$, if and only if $\delta_{x}(U)=1$.

For every continuous valuation $\nu$ on a space $X$, let $\operatorname{Val}(\nu)$ denote the set of non-trivial values $\{\nu(U) \mid U \in \mathcal{O} X, \nu(U) \neq 0,+\infty\}$ taken by $\nu$. 
Lemma 4.2. Let $\nu$ be a continuous valuation on a space $X$, with the property that $\operatorname{Val}(\nu)$ has a least element $r$. Then there is an irreducible closed subset $C$ of $X$ such that:

(1) $\nu^{\prime} \stackrel{\text { def }}{=} \nu-r \mathfrak{e}_{C}$ is a continuous valuation;

(2) $\operatorname{Val}\left(\nu^{\prime}\right) \subseteq\{v-r \mid v \in \operatorname{Val}(\nu), v \neq r\}$.

Proof. Let $U_{r}$ be any open subset of $X$ such that $\nu\left(U_{r}\right)=r \neq 0$. We consider the family $\mathcal{U}$ of open subsets $U$ of $X$ such that $\nu\left(U \cap U_{r}\right)=0$, or equivalently $\nu\left(U \cap U_{r}\right)<r$. That family contains the empty set, and for any two elements $U, V$ of $\mathcal{U}$, we have $\nu\left((U \cup V) \cap U_{r}\right) \leq \nu\left(U \cap U_{r}\right)+\nu\left(V \cap U_{r}\right)=0$, so $U \cup V$ is in $\mathcal{U}$. It follows that $\mathcal{U}$ is directed. Let $U_{*}$ be the union of all

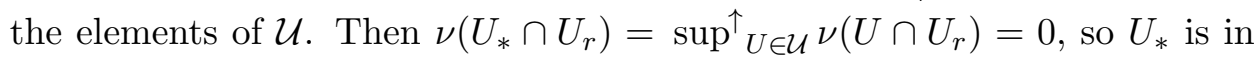
$\mathcal{U}$. It follows that $U_{*}$ is the largest element of $\mathcal{U}$. In particular, for every $U \in \mathcal{O} X, \nu\left(U \cap U_{r}\right)=0$ if and only if $U \subseteq U_{*}$.

We define $C$ as the complement of $U_{*}$. Since $\nu\left(U_{r}\right) \neq 0, U_{r}$ is not included in $U_{*}$, so $C$ intersects $U_{r}$. In particular, $C$ is non-empty. For any two open subsets $U$ and $V$ of $X$ that intersect $C$, we claim that $U \cap V$ also intersects $C$. This will show that $C$ is irreducible. By assumption, neither $U$ nor $V$ is included in $U_{*}$, so $\nu\left(U \cap U_{r}\right) \geq r$ and $\nu\left(V \cap U_{r}\right) \geq r$. It follows that $\nu\left((U \cap V) \cap U_{r}\right)=\nu\left(U \cap U_{r}\right)+\nu\left(V \cap U_{r}\right)-\nu\left((U \cup V) \cap U_{r}\right) \geq 2 r-r=r$, using modularity and the inequality $\nu\left((U \cup V) \cap U_{r}\right) \leq \nu\left(U_{r}\right)=r$. We conclude that $U \cap V$ is not included in $U_{*}$, hence intersects $C$.

(1) Let $\nu^{\prime} \stackrel{\text { def }}{=} \nu-r \mathfrak{e}_{C}$. We first verify that $\nu^{\prime}$ is monotonic. Let $U \subseteq V$ be two open subsets of $X$. If $\mathfrak{e}_{C}(U)=\mathfrak{e}_{C}(V)$, then $\nu^{\prime}(U) \leq \nu^{\prime}(V)$. Therefore let us assume that $\mathfrak{e}_{C}(U) \neq \mathfrak{e}_{C}(V)$, hence necessarily $\mathfrak{e}_{C}(U)=0, \mathfrak{e}_{C}(V)=1$. As a consequence, $\nu\left(U \cap U_{r}\right)=0$ and $\nu\left(V \cap U_{r}\right) \geq r$. Then:

$$
\begin{aligned}
\nu^{\prime}(U)=\nu(U) & =\nu\left(U \cup U_{r}\right)+\nu\left(U \cap U_{r}\right)-\nu\left(U_{r}\right) \\
& \text { by modularity, and since } \nu\left(U_{r}\right)<+\infty \\
& =\nu\left(U \cup U_{r}\right)-r \\
& \leq \nu\left(V \cup U_{r}\right)-r \\
& =\nu(V)+\nu\left(U_{r}\right)-\nu\left(V \cap U_{r}\right)-r
\end{aligned}
$$

by modularity, and since $\nu\left(V \cap U_{r}\right) \leq \nu\left(U_{r}\right)<+\infty$

$$
\leq \nu(V)+\nu\left(U_{r}\right)-r-r=\nu^{\prime}(V) \text {. }
$$

In particular, since $\nu^{\prime}(\emptyset)=0, \nu^{\prime}$ takes its values in $\overline{\mathbb{R}}_{+}$. It then follows from Lemma 3.5 that $\nu^{\prime}$ is a continuous valuation. 
(2) Let $V^{\prime} \stackrel{\text { def }}{=}\{v-r \mid v \in \operatorname{Val}(\nu), v \neq r\}$. For every $U \in \mathcal{O} X$, if $U$ intersects $C$ then $\nu^{\prime}(U)=\nu(U)-r$; hence if $\nu^{\prime}(U) \neq 0,+\infty$, then $\nu^{\prime}(U) \in V^{\prime}$. Otherwise, $U \subseteq U_{*}$, so $\nu\left(U \cap U_{r}\right)=0$, and therefore $\nu^{\prime}(U)=\nu(U)=$ $\nu\left(U \cup U_{r}\right)+\nu\left(U \cap U_{r}\right)-\nu\left(U_{r}\right)=\nu\left(U \cup U_{r}\right)-r$, using modularity and the fact that $\nu\left(U_{r}\right)=r<+\infty$. Hence if $\nu^{\prime}(U) \neq 0,+\infty$, then $\nu^{\prime}(U)$ is in $V^{\prime}$.

Proposition 4.3. Let $X$ be a topological space. The bounded continuous valuations $\nu$ on $X$ that take only finitely many values are exactly the finite linear combinations $\sum_{i=1}^{n} a_{i} \mathfrak{e}_{C_{i}}$, where each $C_{i}$ is irreducible closed and $a_{i} \in$ $\mathbb{R}_{+} \backslash\{0\}$.

Proof. That $\sum_{i=1}^{n} a_{i} \mathfrak{e}_{C_{i}}$ only takes finitely many values is obvious. We prove the converse implication by induction on the number $n$ of non-zero values taken by $\nu$. If $n=0$, then $\nu$ is the zero valuation. Otherwise, let $r$ be the least non-zero value taken by $\nu$. We find $C$ and $\nu^{\prime}$ as in Lemma 4.2 . Since $\operatorname{Val}\left(\nu^{\prime}\right)$ has one less element than $\operatorname{Val}(\nu)$, we can apply the induction hypothesis, allowing us to conclude.

Proposition 4.4. Every continuous valuation $\nu$ on a topological space $X$ that takes only finitely many distinct values is point-continuous.

Proof. If $\nu$ is bounded, then by Proposition 4.3, $\nu$ is of the form $\sum_{i=1} a_{i} \mathfrak{e}_{i}$, where each $C_{i}$ is irreducible closed and $a_{i} \in \mathbb{R}_{+}$, and $\mathfrak{e}_{C_{i}}$ is point-continuous. Any linear combination of point-continuous valuations is point-continuous [11, Section 3.2], so $\nu$ is point-continuous.

Hence we concentrate on the case where $\nu(X)$ is equal to $+\infty$. Let $s$ be the greatest finite value that $\nu$ takes. Consider the family $\mathcal{S}$ of all opens $U$ such that $\nu(U) \leq s$. For any $U, V \in \mathcal{S}, \nu(U \cup V)=\nu(U)+\nu(V)-\nu(U \cap V)$, which is obviously a finite value. Hence $U \cup V$ is in $S$. This implies that the family $S$ is directed. Let $U_{s}$ be the directed union of $\mathcal{S}$, and $C_{\infty}$ be the complement of $U_{s}$. We notice that $\nu\left(U_{s}\right)=\sup _{U \in \mathcal{S}} \nu(U)=s$, and hence that $U_{s}$ is a proper subset of $X$.

The collection $\mathcal{F}$ of simple valuations $\sum_{i=1}^{n} a_{i} \delta_{x_{i}}$ such that every $x_{i}$ is in $C_{\infty}$ is directed: it is non-empty because $C_{\infty}$ is non-empty, and any two elements $\sum_{i=1}^{n} a_{i} \delta_{x_{i}}$ and $\sum_{i=1}^{n} b_{i} \delta_{x_{i}}$ (which we take over the same set of points $x_{i}$, without loss of generality), have an upper bound, such as $\sum_{i=1}^{n} \max \left(a_{i}, b_{i}\right) \delta_{x_{i}}$. Let $\mu$ be the supremum of $\mathcal{F}$. Since the family of pointcontinuous valuations is closed under directed suprema [11, Section 3.2 (5)], $\mu$ is point-continuous.

We compute $\mu$ explicitly. For every open subset $U$ of $X$, if $U \subseteq U_{s}$, namely if $U$ and $C_{\infty}$ are disjoint, then the value of any element $\sum_{i=1}^{n} a_{i} \delta_{x_{i}}$ 
of $\mathcal{F}$ on $U$ is zero, so $\mu(U)=0$. Otherwise, let us pick an element $x$ from $U \cap C_{\infty}$. Then $a \delta_{x}$ is in $\mathcal{F}$ for every $a \in \mathbb{R}_{+}$, so $\mu(U) \geq a \delta_{x}(U)=a$ for every $a \in \mathbb{R}_{+}$, from which it follows that $\mu(U)=+\infty$.

Let $\nu_{\mid U_{s}}$ be the restriction of $\nu$ to $U_{s}$, namely the map $V \mapsto \nu\left(U_{s} \cap\right.$ $V)$. This is a continuous valuation [11, Section 3.3]. It is bounded by construction of $U_{s}$, and takes only finitely many values. Therefore, as we have already seen, Proposition 4.3 entails that $\nu_{\mid U_{s}}$ is point-continuous.

We now observe that $\nu=\nu_{\mid U_{s}}+\mu$. For every $U \in \mathcal{O} X$, if $U \subseteq U_{s}$, then $\nu_{\mid U_{s}}(U)+\mu(U)=\nu\left(U \cap U_{s}\right)+0=\nu(U)$. Otherwise $\nu_{\mid U_{s}}(U)+\mu(U)=$ $\nu_{\mid U_{s}}(U)+(+\infty)=+\infty=\nu(U)$. Being a sum of two point-continuous valuations, $\nu$ is point-continuous.

\subsection{The Sorgenfrey line}

Let $\mathbb{R}$ be the set of real numbers, with its usual metric topology.

The Sorgenfrey line $\mathbb{R}_{\ell}$ has the same set of points as $\mathbb{R}$, but its topology is generated by the half-open intervals $\left[a, b\left[, a<b\left[23\right.\right.\right.$. The topology of $\mathbb{R}_{\ell}$ is finer than that of $\mathbb{R}$. $\mathbb{R}_{\ell}$ is a zero-dimensional, first-countable space [8, Exercices 4.1.34, 4.7.17]. It is paracompact hence $T_{4}$, and Choquet-complete hence a Baire space [8, Exercises $6.3 .32,7.6 .11] . \mathbb{R}_{\ell}$ is not locally compact, as every compact subset of $\mathbb{R}_{\ell}$ has empty interior [8, Exercise 4.8.5]. In fact, $\mathbb{R}_{\ell}$ is not even consonant [4, 5]. Although it is first-countable, $\mathbb{R}_{\ell}$ is not second-countable [8, Exercise 6.3.10].

A hereditarily Lindelöf space is a space in which every family $\left(U_{i}\right)_{i \in I}$ of open subsets has a countable subfamily with the same union, or equivalently a space whose subspaces are all Lindelöf. Every second-countable space is hereditarily Lindelöf, but $\mathbb{R}_{\ell}$ is a counterexample to the reverse implication, as the following folklore result demonstrates.

Proposition 4.5. $\mathbb{R}_{\ell}$ is hereditarily Lindelöf.

Proof. Let us denote by $\stackrel{\circ}{U}$ the interior of any set $U$ in the topology of $\mathbb{R}$ $\left(\right.$ not $\mathbb{R}_{\ell}$ ).

Let $\left(U_{i}\right)_{i \in I}$ be any family of open subsets of $\mathbb{R}_{\ell}, U \stackrel{\text { def }}{=} \bigcup_{i \in I} U_{i}$ and $U^{\prime} \stackrel{\text { def }}{=}$ $\bigcup_{i \in I} \stackrel{\circ}{U}_{i}$. Since $\mathbb{R}$ is second-countable hence hereditarily Lindelöf, there is a countable subset $J$ of $I$ such that $U^{\prime}=\bigcup_{i \in J} \stackrel{\circ}{U}_{j}$.

We claim that $U \backslash U^{\prime}$ is countable. For each point $x \in U \backslash U^{\prime}, x$ is in some $U_{i}$ hence in some basic open set $\left[a, b\left[\subseteq U_{i}\right.\right.$. Then $x$ is also included in the smaller basic open set $\left[x, b\left[\right.\right.$. Let us write $b$ as $x+\delta_{x}$, with $\delta_{x}>0$. We observe that $] x, x+\delta_{x}\left[\right.$ is included in $\stackrel{\circ}{U}_{i}$, hence in $U^{\prime}$. 
Let $x$ and $y$ be two points of $U \backslash U^{\prime}$, with $x<y$. If $\left[x, x+\delta_{x}\right.$ [ and $\left[y, y+\delta_{y}\left[\right.\right.$ intersect, then $y$ is in $\left[x, x+\delta_{x}[\right.$, and since $x \neq y$, it follows that $y$ is in $] x, x+\delta_{x}$ [. That is impossible, since ] $x, x+\delta_{x}$ [ is included in $U^{\prime}$ and $y$ is not in $U^{\prime}$.

Therefore, for any two distinct points $x$ and $y$ of $U \backslash U^{\prime},\left[x, x+\delta_{x}[\right.$ and $\left[y, y+\delta_{y}\left[\right.\right.$ are disjoint. We pick one rational number $q_{x}$ in each set $\left[x, x+\delta_{x}[\right.$ with $x \in U \backslash U^{\prime}$ : then $x \neq y$ implies $q_{x} \neq q_{y}$, and therefore $U \backslash U^{\prime}$ is countable.

Let us now pick an index $i_{x} \in I$ such that $x \in U_{i_{x}}$, one for each $x \in U \backslash$ $U^{\prime}$. Then $U=U^{\prime} \cup\left(U \backslash U^{\prime}\right) \subseteq U^{\prime} \cup \bigcup_{x \in U \backslash U^{\prime}} U_{i_{x}} \subseteq U$, so $\left(U_{i}\right)_{i \in J \cup\left\{i_{x} \mid x \in U \backslash U^{\prime}\right\}}$ is a countable subfamily with the same union as our original family $\left(U_{i}\right)_{i \in I}$.

Lemma 4.6. $\mathbb{R}$ and $\mathbb{R}_{\ell}$ have the same Borel $\sigma$-algebra.

Proof. Let $\Sigma$ be the Borel $\sigma$-algebra of $\mathbb{R}$, and $\Sigma_{\ell}$ be that of $\mathbb{R}_{\ell}$. Clearly, $\Sigma \subseteq \Sigma_{\ell}$

In the converse direction, we first claim that every open subset $U$ of $\mathbb{R}_{\ell}$ is in $\Sigma$. For every $x \in U$, the union of all the intervals included in $U$ and containing $x$ is convex, hence is an interval $I_{x}$. Let $a_{x}$ be its lower end, $b_{x}$ be its upper end. We note that $b_{x}$ cannot be in $I_{x}$, otherwise $b_{x}$ would be in some basic open subset $\left[b_{x}, b_{x}+\delta_{x}\right.$ [ included in $U$, which would allow us to form a strictly larger interval included in $U$ and containing $x$. Hence $I_{x}$ is equal to $\left[a_{x}, b_{x}[\right.$ or to $] a_{x}, b_{x}\left[\right.$. In any case, $a_{x}<b_{x}$, so $I_{x}$ contains a rational number $q_{x}$. Since the intervals $I_{x}$ are pairwise disjoint, there are no more such intervals than there are rational numbers. Moreover, $\bigcup_{x \in U} I_{x}$ is equal to $U$. It follows that $U$ is a countable union of pairwise disjoint intervals, and is therefore in $\Sigma$.

Since $\Sigma_{\ell}$ is the smallest $\sigma$-algebra containing the open subsets of $\mathbb{R}_{\ell}$, we conclude that $\Sigma_{\ell} \subseteq \Sigma$.

Given a topological space $X$, a Borel measure on $X$ is $\tau$-smooth if and only if its restriction to the lattice $\mathcal{O} X$ of open subsets of $X$ is a continuous valuation. Adamski showed that a space $X$ is hereditarily Lindelöf if and only if every Borel measure on $X$ is $\tau$-smooth [2, Theorem 3.1].

Proposition 4.7. For every Borel measure $\mu$ on $\mathbb{R}$, the restriction of $\mu$ to the open subsets of $\mathbb{R}_{\ell}$ is a continuous valuation.

Proof. By Lemma 4.6, $\mu$ is also a Borel measure on $\mathbb{R}_{\ell}$. We then apply Adamski's theorem, thanks to Proposition 4.5. 
Corollary 4.8. The restriction $\lambda$ of the Lebesgue measure on $\mathbb{R}$ to $\mathcal{O} \mathbb{R}_{\ell}$ is a continuous valuation.

\subsection{The compact subsets of $\mathbb{R}_{\ell}$}

We recall that every compact subset $Q$ of $\mathbb{R}_{\ell}$ has empty interior. For completeness, we give the proof here. Let us assume a compact subset $Q$ of $\mathbb{R}_{\ell}$ with non-empty interior. $Q$ contains a basic open set $[a, b[$ with $a<b$. $[a, b[$ is not only open, but also closed, since it is the complement of the open set $]-\infty, a[\cup[b,+\infty[$. (Those two sets are open, being equal to $\bigcup_{m \in \mathbb{N}}\left[a-m-1, a-m\left[\right.\right.$ and to $\bigcup_{n \in \mathbb{N}}[b+n, b+n+1[$ respectively). Being closed in a compact set, $\left[a, b\left[\right.\right.$ is compact. But the open cover $\left(\left[a, b-\epsilon[)_{\epsilon \in] 0, b-a[}\right.\right.$ of $[a, b[$ has no finite subcover: contradiction.

We use the following folklore result. The fact that every compact subset of $\mathbb{R}_{\ell}$ is countable is the only thing we will need to know in later sections, together with Corollary 4.14 below, but we think that giving a complete characterization of the compact subsets of $\mathbb{R}_{\ell}$ is interesting in its own right, and may help one understand better what they look like.

Lemma 4.9. Every compact subset $Q$ of $\mathbb{R}_{\ell}$ is countable, bounded, and is well-founded in the ordering $\geq$.

Proof. For every point $x$ of $Q$, the family of open sets $]-\infty, x-\epsilon[(\epsilon>0)$ plus $\left[x,+\infty\left[\right.\right.$ is an open cover of $Q$ (in fact, of the whole of $\mathbb{R}_{\ell}$ ), hence contains a finite subcover. It follows that $Q$ is contained in $]-\infty, x-\epsilon_{x}[\cup[x,+\infty[$ for some $\epsilon_{x}>0$. Equivalently, $Q$ contains no point in $\left[x-\epsilon_{x}, x[\right.$.

From this, we deduce that $\left[x-\epsilon_{x}, x\left[\right.\right.$ and $\left[y-\epsilon_{y}, y[\right.$ are disjoint for any two distinct points $x$ and $y$ of $Q$. Indeed, without loss of generality, let us assume that $y<x$. Since $y$ is in $Q$, it is not in $\left[x-\epsilon_{x}, x\right.$ [, so $y>x$ (which is impossible), or $y \leq x-\epsilon_{x}$. Then $\left[y-\epsilon_{y}, y\right.$ [ lies entirely to the left of $\left[x-\epsilon_{x}, x[\right.$, and does not intersect it.

Next, for each $x \in Q$, we pick a rational number $q_{x}$ in $\left[x-\epsilon_{x}, x[\right.$. By the disjointness property we just proved, the map $x \in Q \mapsto q_{x}$ is injective, so $Q$ is countable.

Since $Q$ is compact in $\mathbb{R}_{\ell}$ and the topology of $\mathbb{R}_{\ell}$ is finer than that of $\overline{\mathbb{R}}_{+}, Q$ is also compact in $\mathbb{R}$, hence is bounded.

Let us imagine that $Q$ contains an infinite increasing sequence $r_{0}<r_{1}<$ $\cdots<r_{n}<\cdots$. Let also $r \stackrel{\text { def }}{=} \sup _{n \in \mathbb{N}}^{\uparrow} r_{n}$. Then the open sets $]-\infty, r_{0}[$, $\left[r_{0}, r_{1}\left[, \ldots,\left[r_{n}, r_{n+1}[, \ldots\right.\right.\right.$, and $[r,+\infty[$ (if $r<+\infty$, otherwise we ignore the last interval) form an open cover of $Q$ without a finite subcover. It follows that $Q$ is well-founded in the ordering $\geq$. 
We now give a few seemingly lesser known results. Given a compact subset $Q$ of $\mathbb{R}_{\ell}, Q$ is a chain, namely a totally ordered subset of $(\mathbb{R}, \geq)$. Notice that we use the reverse ordering $\geq$ : Lemma 4.9 tells us that $Q$ is even a well-founded chain. A chain $A$ in a poset $(P, \sqsubseteq)$ is a subdcpo of $P$ if and only if for every non-empty (equivalently, directed) subset $D$ of $A$, the supremum of $D$ exists in $P$ and is in $A$.

Lemma 4.10. Every compact subset $Q$ of $\mathbb{R}_{\ell}$ is a subdcpo of $(\mathbb{R}, \geq)$ : for every non-empty subset $D$ of $Q$, $\inf D$ is in $Q$. If $Q$ is non-empty, then $Q$ has a least element in the usual ordering $\leq$.

Proof. We first need to note that a net $\left(x_{i}\right)_{i \in I, \sqsubseteq}$ converges to $x$ in $\mathbb{R}_{\ell}$ if and only if $x_{i}$ tends to $x$ from the right, namely: for every $\epsilon>0, x \leq x_{i}<x+\epsilon$ for $i$ large enough [8, Exercise 4.7.6].

Let $D$ be any non-empty subset of $Q$, and let $r \stackrel{\text { def }}{=} \inf D$. By Lemma 4.9 , $Q$ is bounded, so $r$ is a well-defined real number. Since $Q$ is well-founded, $D$ is isomorphic to a unique ordinal $\beta$, and we can write the elements of $D$ as $r_{\alpha}, \alpha<\beta$, in such a way that for all $\alpha, \alpha^{\prime}<\beta, \alpha \leq \alpha^{\prime}$ if and only if $r_{\alpha} \geq r_{\alpha^{\prime}}$. The net $\left(r_{\alpha}\right)_{\alpha<\beta, \leq}$ then converges to $r$ from the right. Since $\mathbb{R}_{\ell}$ is $T_{2}$, its compact subset $Q$ is closed, so $r$ is in $Q$.

If $Q$ is non-empty, we can take $D \stackrel{\text { def }}{=} Q$. Then $\inf D \in Q$ is the least element of $Q$.

Another way of expressing Lemma 4.10 , together with the well-foundedness property of Lemma 4.9 is to say the following. The fact that $\beta_{Q}$ is not a limit ordinal is due to the fact that $Q$ must be empty or have a least element, namely that $\beta_{Q}$ must be equal to 0 or have a largest element.

Lemma 4.11. For every compact subset $Q$ of $\mathbb{R}_{\ell},(Q, \geq)$ is order-isomorphic to a unique ordinal $\beta_{Q} ; \beta_{Q}$ is not a limit ordinal, and the order-isomorphism is a Scott-continuous map from $\beta_{Q}$ to $(Q, \geq)$.

Lemma 4.12. Let $\beta$ be a non-limit ordinal, and $f: \beta \rightarrow(\mathbb{R}, \geq)$ be a Scottcontinuous map. The image $\operatorname{Im} f$ of $f$ is compact in $\mathbb{R}_{\ell}$.

Proof. Since $\beta$ is a non-limit ordinal, it is a dcpo. Its finite elements are the ordinals $\alpha<\beta$ that are not limit ordinals, and then it is easy to see that $\beta$ is an algebraic domain. It is also a complete lattice, and every algebraic complete lattice is Lawson-compact [6, Corollary III.1.11].

We claim that $f$ is continuous from $\beta$, with its Lawson topology, to $\mathbb{R}_{\ell}$. Let $\left[a, b\left[\right.\right.$ be any subbasic open subset of $\mathbb{R}_{\ell}$. We aim to show that $f^{-1}([a, b[)$ is Lawson-open. If $f^{-1}(]-\infty, a[)$ is empty, then $f^{-1}\left(\left[a, b[)=f^{-1}(]-\infty, b[)\right.\right.$, 
which is Scott-open since $f$ is Scott-continuous; in particular it is Lawsonopen. So let us assume that $f^{-1}(]-\infty, a[)$ is non-empty. Since $\beta$ is wellfounded, $f^{-1}(]-\infty, a[)$ has a least element $\alpha$. Then $f^{-1}(]-\infty, a[)=\uparrow \alpha$, and therefore $f^{-1}\left(\left[a, b[)=f^{-1}(]-\infty, b[) \backslash f^{-1}(]-\infty, a[)=f^{-1}(]-\infty, b[) \backslash \uparrow \alpha\right.\right.$ is Lawson-open.

Since $\beta$ is compact in the Lawson topology, it follows that $\operatorname{Im} f$ is compact in $\mathbb{R}_{\ell}$.

In particular, we obtain the following converse to Lemma 4.10.

Lemma 4.13. Every well-founded subdcpo $Q$ of $(\mathbb{R}, \geq)$ is compact in $\mathbb{R}_{\ell}$.

Proof. $Q$ is order-isomorphic to a unique ordinal $\beta_{Q}$ through some map $f: \beta_{Q} \rightarrow(Q, \geq)$. Since $Q$ has a least element, $\beta_{Q}$ has a largest element, so $\beta_{Q}$ cannot be a limit ordinal. Let $D$ be any non-empty family $D$ in $\beta_{Q}$. Since $Q$ is a subdcpo of $(\mathbb{R}, \geq)$, $\inf f(D)$ is an element $f(\alpha)$ of $Q$. Since $f$ is an order-isomorphism, $\alpha$ is the supremum of $D$, and therefore $f$ is Scott-continuous. We can now apply Lemma 4.12 .

Corollary 4.14. Let $x$ be any real number and $x_{0}>x_{1}>\cdots>x_{n} \cdots$ be any decreasing sequence of real numbers such that $\inf _{n \in \mathbb{N}}^{\downarrow} x_{n}=x$. Then $\left\{x_{0}, x_{1}, \cdots, x_{n}, \cdots\right\} \cup\{x\}$ is compact in $\mathbb{R}_{\ell}$.

Together, Lemmas 4.9, 4.11, and 4.13 imply the following.

Theorem 4.15. The compact subsets of $\mathbb{R}_{\ell}$ are exactly the well-founded subdcpos of $(\mathbb{R}, \geq)$. They are all countable.

Remark 4.16. In general, any well-founded chain in $(\mathbb{R}, \geq)$ is countable. Conversely, for every countable ordinal $\beta$, there is a well-founded chain of $(\mathbb{R}, \geq)$ that is order-isomorphic to $\beta$. This is proved by induction on $\beta$, using the fact that every countable ordinal has countable cofinality, namely is the supremum of countably many strictly lower countable ordinals. All this is folklore, and is left as an exercise.

Remark 4.17. A space $X$ is consonant if and only if, for every Scott-open subset $\mathcal{U}$ of $\mathcal{O} X$, for every $U \in \mathcal{U}$, there is a compact saturated subset $Q$ of $X$ such that $U \in \mathbf{\square} Q \mathcal{U}$, where $Q$ is the set of open neighborhoods of $Q$. We now have enough to give an elementary proof that $\mathbb{R}_{\ell}$ is not consonant [4, 5]. Let us pick any real number $r>0$. By Corollary $\left.\left.4.8, \mathcal{U} \stackrel{\text { def }}{=} \lambda^{-1}(] r,+\infty\right]\right)$ is a Scott-open subset of $\mathcal{O} \mathbb{R}_{\ell}$. For every compact (saturated) subset $Q$ of $\mathbb{R}_{\ell}$, $Q$ is countable. Let us write $Q$ as $\left\{x_{n} \mid n \in I\right\}$, where $I$ is some subset of $\mathbb{N}$. 
Then $V \stackrel{\text { def }}{=} \bigcup_{n \in I}\left[x_{n}, x_{n}+r / 2^{n+1}[\right.$ is an open neighborhood of $Q$ such that $\lambda(V) \leq \sum_{n \in I} r / 2^{n+1} \leq r$, so $V$ is not in $\mathcal{U}$. It follows that no set of the form $Q$ is included in $\mathcal{U}$.

\subsection{The dcpo $\mathcal{Q} \mathbb{R}_{\ell}$}

Given any topological space $X$, we can form the set $\mathcal{Q} X$ of all non-empty compact saturated subsets of $X . \mathcal{Q} X$ is a poset under the reverse inclusion ordering $\supseteq$ called the Smyth powerdomain of $X$. When $X$ is well-filtered, this is a dcpo, where suprema of directed families $\left(Q_{i}\right)_{i \in I}$ are their intersection $\bigcap_{i \in I}^{\downarrow} Q_{i}\left[8\right.$, Proposition 8.3.25]. This is notably the case when $X=\mathbb{R}_{\ell}$, since $\mathbb{R}_{\ell}$ is $T_{2}$, hence sober, hence well-filtered. Note also that, in that case, every subset is saturated, so we may safely omit "saturated" from the description of elements of $\mathcal{Q} \mathbb{R}_{\ell}$.

There are at least two topologies of interest on $\mathcal{Q} X$. One is the Scott topology on the poset $(\mathcal{Q} X, \supseteq)$. Another one is the upper Vietoris topology, whose basic open sets are the sets $\square U \stackrel{\text { def }}{=}\{Q \in \mathcal{Q} X \mid Q \subseteq U\}$, where $U$ ranges over the open subsets of $X$. When $X$ is well-filtered (e.g., if $\left.X=\mathbb{R}_{\ell}\right), \square U$ is Scott-open, and hence the Scott topology is finer than the upper Vietoris topology.

The set $\operatorname{Max} \mathcal{Q} \mathbb{R}_{\ell}$ of maximal points of $\mathcal{Q} \mathbb{R}_{\ell}$ consists of the one-element compact sets $\{x\}, x \in \mathbb{R}$. By equating them with $x$, we equate the set $\operatorname{Max} \mathcal{Q} \mathbb{R}_{\ell}$ with $\mathbb{R}_{\ell}$. This allows us to write $\mathcal{U} \cap \mathbb{R}_{\ell}$ for any subset $\mathcal{U}$ of $\mathcal{Q} \mathbb{R}_{\ell}$.

Lemma 4.18. For every Scott-open subset $\mathcal{U}$ of $\mathcal{Q} \mathbb{R}_{\ell}, \mathcal{U} \cap \mathbb{R}_{\ell}$ is open in $\mathbb{R}_{\ell}$.

Proof. Let $x$ be an arbitrary point of $\mathcal{U} \cap \mathbb{R}_{\ell}$. We claim that $\mathcal{U} \cap \mathbb{R}_{\ell}$ contains an interval $[x, x+\epsilon$ for some $\epsilon>0$. We reason by contradiction, and we assume that every interval $\left[x, x+\epsilon\right.$ [ contains a point outside $\mathcal{U} \cap \mathbb{R}_{\ell}$.

We use this to build a sequence of points $x_{0}>x_{1}>\cdots>x_{n}>\cdots \geq x$ as follows. Since $\mathcal{U} \cap \mathbb{R}_{\ell}$ does not contain $\left[x, x+1\right.$ [, there is a point $x_{0}$ in $\left[x, x+1\left[\right.\right.$ that is not in $\mathcal{U} \in \mathbb{R}_{\ell}$. This cannot be $x$, since $x$ is in $\mathcal{U} \cap \mathbb{R}_{\ell}$. Hence $\min \left(x_{0}, x+1 / 2\right)>x$. Since $\mathcal{U} \cap \mathbb{R}_{\ell}$ does not contain $\left[x, \min \left(x_{0}, x+1 / 2\right)[\right.$, there is a point $x_{1}$ in $\left[x, \min \left(x_{0}, x+1 / 2\right)\right.$ [ that is not in $\mathcal{U} \in \mathbb{R}_{\ell}$. Again, $x_{1}$ is different from $x$. This allows us to build the interval $\left[x, \min \left(x_{1}, x+1 / 4\right)\right.$, and as before, this must contain a point $x_{2}$ outside $\mathcal{U} \cap \mathbb{R}_{\ell}$. By induction, this allows us to define points $x_{n}$ outside $\mathcal{U} \cap \mathbb{R}_{\ell}$ such that $x_{n+1} \in\left[x, \min \left(x_{n}, x+\right.\right.$ $\left.1 / 2^{n+1}\right)\left[\right.$. In particular, $x_{0}>x_{1}>\cdots>x_{n}>\cdots \geq x$. Also, inf ${ }_{n \in \mathbb{N}} x_{n}=$ $x$. 
For each $n \in \mathbb{N}$, let $Q_{n}$ be the set $\left\{x_{m} \mid m \geq n\right\} \cup\{x\}$. This is compact in $\mathbb{R}_{\ell}$ by Corollary 4.14 .

It is clear that $Q_{n}$ is non-empty, and that $\bigcap_{n \in \mathbb{N}}^{\downarrow} Q_{n}=\{x\}$, which is in $\mathcal{U}$. Since $\mathcal{U}$ is Scott-open, some $Q_{n}$ must be in $\mathcal{U}$. This implies that $\left\{x_{n}\right\}$, which is included, hence above $Q_{n}$ in $\mathcal{Q R} \mathbb{R}_{\ell}$, is also in $\mathcal{U}$. Therefore $x_{n}$ is in $\mathcal{U} \cap \mathbb{R}_{\ell}$; but that is impossible, since all the points $x_{n}$ were built so as to lie outside $\mathcal{U} \cap \mathbb{R}_{\ell}$.

A dcpo model of a $T_{1}$ space $X$ is a dcpo $P$ such that Max $P$, the subset of maximal elements of $P$ with the subspace topology, from the Scott topology on $P$, is homeomorphic to $X$. The following is a special case of Corollary 2.12 of [10, which says that for every $T_{1}$, first-countable and well-filtered space, $\mathcal{Q} X$ is a dcpo model of $X$.

Theorem 4.19. $\mathcal{Q} \mathbb{R}_{\ell}$ is a dcpo model of $\mathbb{R}_{\ell}$.

Proof. By Lemma 4.18, every open subset in the subspace topology on $\operatorname{Max} \mathcal{Q} \mathbb{R}_{\ell}$ is open in $\mathbb{R}_{\ell}$. Conversely, for every open subset $U$ of $\mathbb{R}_{\ell}, \square U$ is a Scott-open subset of $\mathcal{Q} \mathbb{R}_{\ell}$ whose intersection with $\mathbb{R}_{\ell}$ is equal to $U$, so $U$ is open in the subspace topology on $\operatorname{Max} \mathcal{Q} \mathbb{R}_{\ell}$.

Remark 4.20. Actually, one can expect more in this case. Xu and Yang[26] proved that for a first-countable well-filtered space $X$, in which each compact saturated subset has countable minimal elements (in the specialization order), the Scott and the upper Vietoris topologies coincide on $\mathcal{Q} X$. As we have seen from above that $\mathbb{R}_{\ell}$ does satisfy these properties, hence the Scott and the upper Vietoris topologies coincide on $\mathcal{Q} \mathbb{R}_{\ell}$. This is slightly stronger than Theorem 4.19. We speak in full of the development of Theorem 4.19 in order to keep this note self-contained.

\subsection{A continuous, non point-continuous valuation on $\mathcal{Q} \mathbb{R}_{\ell}$}

By Proposition 4.7, every Borel measure $\mu$ on $\mathbb{R}_{\ell}$ defines a continuous valuation by restriction to $\mathcal{O} \mathbb{R}_{\ell}$, and we again write that continuous valuation as $\mu$. By Theorem 4.19, the map $x \mapsto\{x\}$ is a topological embedding of $\mathbb{R}_{\ell}$ into $\mathcal{Q} \mathbb{R}_{\ell}$. The image of $\mu$ by that embedding is a continuous valuation $\bar{\mu}$ on $\mathcal{Q} \mathbb{R}_{\ell}$. Explicitly, we have:

$$
\bar{\mu}(\mathcal{U}) \stackrel{\text { def }}{=} \mu\left(\mathcal{U} \cap \mathbb{R}_{\ell}\right)
$$

for every Scott-open subset $\mathcal{U}$ of $\mathcal{Q} \mathbb{R}_{\ell}$. 
Theorem 4.21. Let $\mu$ be any Borel measure on $\mathbb{R}_{\ell}$ with the property that there is an open subset $U$ of $\mathbb{R}_{\ell}$ such that $0<\mu(U)<+\infty$, and $\mu(\{x\})=0$ for every $x \in U$. Then the continuous valuation $\bar{\mu}$ is not point-continuous. In particular, $\bar{\lambda}$ is not point-continuous.

Proof. Since $\mu(U)>0$ and since $\bar{\mu}(\square U)=\mu(U)$, we can find a real number $r$ such that $0<r<\bar{\mu}(\square U)$.

We will show that, for every finite subset $A \stackrel{\text { def }}{=}\left\{Q_{1}, \cdots, Q_{m}\right\}$ of $\square U$, there is an open neighborhood $\mathcal{V}$ of $A$ such that $\bar{\mu}(\mathcal{V}) \leq r$.

We make the following preliminary claim $(*)$ : for every $x \in U$, for every $a>0$, there is an $\epsilon>0$ such that $[x, x+\epsilon[\subseteq U$ and $\mu([x, x+\epsilon[) \leq a$. Indeed, it is a general property of measures that $\mu\left(\bigcap_{n \in \mathbb{N}}^{\downarrow} E_{n}\right)=\inf _{n \in \mathbb{N}}^{\downarrow} \mu\left(E_{n}\right)$ for any decreasing family of measurable subsets $E_{n}$ such that $\mu\left(E_{n}\right)<+\infty$ for at least one $n$. Hence $\mu(\{x\})=\mu\left(\bigcap_{n \in \mathbb{N}}^{\downarrow}\left[x, x+\epsilon_{0} / 2^{n}[)=\right.\right.$ inf $^{\downarrow} n \in \mathbb{N} \mu([x, x+$ $\epsilon_{0} / 2^{n}[)$, where $\epsilon_{0}>0$ is chosen so that $\left[x, x+\epsilon_{0}[\subseteq U\right.$. Since $\mu(\{x\})=0$, there is an $n \in \mathbb{N}$ such that $\mu\left(\left[x, x+\epsilon_{0} / 2^{n}[<a\right.\right.$.

Let $s>0$ be such that $m s \leq r$. For every $i \in\{1, \cdots, m\}, Q_{i}$ is countable (Lemma 4.9), so let us write it as $\left\{x_{i 0}, x_{i 1}, \cdots\right\}$. (We allow for infinite repetitions of elements in order not to have to make a special case when $Q_{i}$ is finite.) For every $i \in\{1, \cdots, m\}$, for every $j \in \mathbb{N}$, we use $(*)$ to find a number $\epsilon_{i j}>0$ such that $\mu\left(\left[x_{i j}, x_{i j}+\epsilon_{i j}[) \leq s / 2^{j+1}\right.\right.$. Let $V_{i} \stackrel{\text { def }}{=} \bigcup_{j \in \mathbb{N}}\left[x_{i j}, x_{i j}+\epsilon_{i j}[\right.$. We note that $\mu\left(V_{i}\right) \leq \sum_{j \in \mathbb{N}} \mu\left(\left[x_{i j}, x_{i j}+\epsilon_{i j}[)=\sum_{j \in \mathbb{N}} s / 2^{j+1}=s\right.\right.$. We now define $V$ as $\bigcup_{i=1}^{m} V_{i}$. Then $\mu(V) \leq \sum_{i=1}^{m} \mu\left(V_{i}\right) \leq m s \leq r$.

Clearly, $Q_{i}$ is included in $V_{i}$, hence in $V$, for every $i \in\{1, \cdots, m\}$, so $A$ is included in $\square V$. We define $\mathcal{V}$ as $\square V$. Then $\bar{\mu}(\mathcal{V})=\mu(V) \leq r$.

\subsection{More remarks on $\mathcal{Q} \mathbb{R}_{\ell}$}

In [21, Theorem 3.1], Lyu and the second author showed that a space $X$ is locally compact if and only if that $\mathcal{Q} X$ is core-compact in the upper Vietoris topology. It is easy to see that $\mathbb{R}_{\ell}$ is not locally compact as the interior of each compact set is empty. So $\mathcal{Q} \mathbb{R}_{\ell}$ is not core-compact in the upper Vietoris topology. By Remark 4.20, we have

Proposition 4.22. $\mathcal{Q} \mathbb{R}_{\ell}$ is not core-compact in its Scott topology.

$\mathbb{R}_{\ell}$ exhibits a diverse mix of pleasant and unpleasant properties, and so does $\mathcal{Q} \mathbb{R}_{\ell}$. While Proposition 4.22 would be on the unpleasant side, the following shows more regularity. 
Proposition 4.23. $\mathcal{Q} \mathbb{R}_{\ell}$ is sober.

Proof. Theorem 3.13 of [13] states that, for any topological space $X, X$ is sober if and only if $\mathcal{Q} X$ is sober in the upper Vietoris topology. Since $\mathbb{R}_{\ell}$ is $T_{2}$, it is sober. By Remark 4.20, the upper Vietoris topology coincides with the Scott topology on $\mathcal{Q} \mathbb{R}_{\ell}$, so $\mathcal{Q} \mathbb{R}_{\ell}$ is sober.

In particular, $\mathcal{Q} \mathbb{R}_{\ell}$ is well-filtered, something we can rederive in another way.

Proposition 4.24. For every well-filtered, coherent space, $\mathcal{Q} X$ is a meetcontinuous dcpo inf-semilattice, which is well-filtered and coherent in its Scott topology. Hence $\mathcal{Q} \mathbb{R}_{\ell}, \mathcal{Q}^{2} \mathbb{R}_{\ell}, \ldots$ all are meet-continuous dcpo infsemilattices, which are well-filtered and coherent in their Scott topologies.

Proof. Let $X$ be a well-filtered, coherent space. In a well-filtered space, filtered intersections $\bigcap_{i \in I}^{\downarrow} Q_{i}$ of compact saturated subsets are compact saturated, and are non-empty if all the sets $Q_{i}$ are non-empty. Hence $\mathcal{Q} X$ is a dcpo.

Given any two elements $Q$ and $Q^{\prime}$ of $\mathcal{Q} X$, their infimum is $Q \cup Q^{\prime}$. Since directed suprema are filtered intersections, and intersections commute with binary unions, $\mathcal{Q} \mathbb{R}_{\ell}$ is a meet-continuous inf-semilattice.

For every dcpo $P$, let its lifting $P_{\perp}$ be $P$ plus a fresh element $\perp$ below all elements of $P$. It is an easy exercise to show that the Scott-open subsets of $P_{\perp}$ are those of $P$, plus $P_{\perp}$ itself, and that the compact saturated subsets of $P_{\perp}$ are those of $P$ plus $P_{\perp}$. It follows that $P$ is well-filtered if and only if $P_{\perp}$ is.

A bounded-complete dcpo is one in which every (upper) bounded family has a least upper bound, or equivalently in which every non-empty family has a greatest lower bound. $\mathcal{Q} X$ is not bounded-complete in general, since the empty family has no least upper bound unless $X$ is compact.

However, $(\mathcal{Q} X)_{\perp}$ is bounded-complete: the least upper bound of the empty set is $\perp$, and the least upper bound of any non-empty set $A$ bounded by some element $Q_{0}$ of $\mathcal{Q} X$ is $\bigcap A$, which is compact because $\mathbb{R}_{\ell}$ is $T_{2}$, and non-empty because it contains $Q_{0}$.

Corollary 3.2 of [25] shows that every bounded-complete dcpo is wellfiltered in its Scott topology. Hence $(\mathcal{Q} X)_{\perp}$ is well-filtered, and therefore $\mathcal{Q} X$ is well-filtered as well.

Lemma 3.1 of [16] states that any well-filtered dcpo $X$ in which $\uparrow x \cap \uparrow y$ is compact saturated for all $x, y \in X$ is coherent. For all $Q, Q^{\prime} \in \mathcal{Q} X$, $\uparrow Q \cap \uparrow Q^{\prime}=\uparrow\left(Q \cap Q^{\prime}\right)$, and $Q \cap Q^{\prime}$ is again in $\mathcal{Q} X$ since $X$ is coherent. In 
particular, $\uparrow Q \cap \uparrow Q^{\prime}$ is a compact saturated subset of $\mathcal{Q} X$. Therefore $\mathcal{Q} X$ is coherent.

Finally, $\mathbb{R}_{\ell}$ is $T_{2}$, hence (sober hence) well-filtered, and coherent, so we may apply the above to $X \stackrel{\text { def }}{=} \mathbb{R}_{\ell}$, then to $X \stackrel{\text { def }}{=} \mathcal{Q} \mathbb{R}_{\ell}$, and so on.

\section{Concluding Remarks}

We have given two concrete examples (Corollary 3.14, Thorem 4.21) on dcpo's to separate minimal valuations, point-continuous valuations and continuous valuations, showing these three classes of valuations differ from each other.

In [17, the Fubini-type equation

$$
\int_{x \in D} \int_{y \in E} h(x, y) d \nu d \xi=\int_{y \in E} \int_{x \in D} h(x, y) d \xi d \nu
$$

is established when either $\nu$ or $\xi$ is point-continuous, where $D$ and $E$ are dcpo's and $h: D \times E \rightarrow \overline{\mathbb{R}}_{+}$are Scott-continuous. (For a definition of the integration, see [18.) This is crucial in proving that Heckmann's pointcontinuous valuations monad is commutative over DCPO. However, it is unknown whether the Equation (2) holds for general continuous valuations $\nu$ and $\xi$, a crucial question in establishing commutativity of the valuations moand $\mathcal{V}$ on DCPO. The aforementioned result in [17] entails that any valuations $\nu$ and $\xi$ that possibly fail Equation (2) must also fail to be pointcontinuous. Hence if one aims to find examples on dcpo's to invalidate Equation (2), the valuations in desire must be non-point-continuous valuations. For the first time, we have given a continuous valuation $\bar{\lambda}$ that is not point-continuous on dcpo's, but more non-point-continuous valuations (of different types from those in Theorem 4.21) are needed before they are sent to test Equation (2).

\section{Acknowledgement}

The second author acknowledges support from NSFC (No. 12001181), and he would also like to thank Andre Kornell and Michael Mislove for useful discussions. 


\section{References}

[1] S. Abramsky and A. Jung. Domain theory. In S. Abramsky, D. M. Gabbay, and T. S. E. Maibaum, editors, Semantic Structures, volume 3 of Handbook of Logic in Computer Science, pages 1-168. Clarendon Press, 1994.

[2] W. Adamski. $\tau$-smooth Borel measures on topological spaces. Mathematische Nachrichten, 78:97-107, 1977.

[3] M. Alvarez-Manilla, A. Edalat, and N. Saheb-Djahromi. An extension result for continuous valuations. Journal of the London Mathematical Society, 61:629-640, 2000.

[4] A. Bouziad. Borel measures in consonant spaces. Topology and its Applications, 70:125-138, 1996.

[5] C. Costantini and S. Watson. On the dissonance of some metrizable spaces. Topology and its Applications, 84:259-268, 1996.

[6] G. Gierz, K. H. Hofmann, K. Keimel, J. D. Lawson, M. Mislove, and D. S. Scott. Continuous Lattices and Domains, volume 93 of Encyclopedia of Mathematics and its Applications. Cambridge University Press, 2003.

[7] J. Goubault-Larrecq. Full abstraction for non-deterministic and probabilistic extensions of PCF I - the angelic cases. Journal of Logical and Algebraic Methods in Programming, 84(1):155-184, 2015.

[8] J. Goubault-Larrecq. Non-Hausdorff Topology and Domain Theory, volume 22 of New Mathematical Monographs. Cambridge University Press, 2013.

[9] J. Goubault-Larrecq. Products and projective limits of continuous valuations on $T_{0}$ spaces. Accepted for publication in "Mathematical Structure in Computer Science", 2021.

[10] Q. He, G. Li, X. Xi, and D. Zhao. Some results on poset models consisting of compact saturated subsets. Electronic Notes in Theoretical Computer Science, 345:77-85, 2019. Proceedings of the 8th International Symposium of Domain Theory (ISDT'19), A. Jung, Q. Li, L. Xu and G.-Q. Zhang, editors. 
[11] R. Heckmann. Spaces of valuations. Technical Report A 09/95, FB 14 Informatik, Universität des Saarlandes, 66041 Saarbrücken, Germany, 1995.

[12] R. Heckmann. Spaces of valuations. In S. Andima, R. C. Flagg, G. Itzkowitz, P. Misra, Y. Kong, and R. Kopperman, editors, Papers on General Topology and Applications: Eleventh Summer Conference at the University of Southern Maine, volume 806 of Annals of the New York Academy of Sciences, pages 174-200, 1996.

[13] R. Heckmann and K. Keimel. Quasicontinuous domains and the Smyth powerdomain. In D. Kozen and M. Mislove, editors, Proceedings of the 29th Conference on the Mathematical Foundations of Programming Semantics, volume 298 of Electronic Notes in Theoretical Computer Science, pages 215-232. Elsevier Science Publishers B.V., 2013.

[14] W. K. Ho and J. Goubault-Larrecq and A. Jung and X. Xi. The HoZhao Problem. Logical Methods in Computer Science, 14(1), 2018.

[15] J. Isbell. Completion of a construction of Johnstone. Proceedings of the American Mathematical Society, 85:333-334, 1982.

[16] X. Jia, A. Jung, and Q. Li. A note on coherence of dcpos. Topology and its Applications, 209:235-238, 2016.

[17] X. Jia, B. Lindenhovius, M. Mislove, and V. Zamdzhiev. Commutative monads for probabilistic programming languages. In Logic in Computer Science (LICS 2021), 2021.

[18] C. Jones. Probabilistic Non-Determinism. PhD thesis, University of Edinburgh, Edinburgh, 1990. Also published as Technical Report No. CST-63-90.

[19] C. Jones and G. Plotkin. A probabilistic powerdomain of evaluations. In Proceedings of the 4th Annual Symposium on Logic in Computer Science, pages 186-195. IEEE Computer Society Press, 1989.

[20] P. T. Johnstone. Scott is not always sober. In Continuous Lattices, Proceedings Bremen, 871:282-283, 1981.

[21] Z. Lyu and X. Jia. Core-compactness of smyth powerspaces. Available at https://arxiv.org/abs/1907.04715, July 2019.

[22] H. L. Royden. Real Analysis. Macmillan, New York, 3rd edition, 1988. 
[23] R. H. Sorgenfrey. On the topological product of paracompact spaces. Bulletin of the American Mathematical Society, 53:631-632, 1947.

[24] R. Tix. Stetige Bewertungen auf topologischen Räumen. Master's thesis, Technische Hochschule Darmstadt, June 1995. 51pp.

[25] X. Xi and J. Lawson. On well-filtered spaces and ordered sets. Topology and its Applications, 228:139-144, September 2017.

[26] X. Xu and Z. Yang. Coincidence of the upper vietoris topology and the scott topology. Topology and its Applications, 288, 107480, December 2021. 